\title{
STUDY ON SCREENING TESTS AMONG THE BLOOD DONORS IN DHAKA MEDICAL COLLEGE HOSPITAL, BANGLADESH
}

\author{
CHOWDHURY FS ${ }^{1}$, BEGUM HA ${ }^{2}$, ADNAN SD ${ }^{3}$, HOQUE MM ${ }^{4}$, HOSSAIN MZ $^{5}$, SIDDIQUI MAE ${ }^{6}$
}

\begin{abstract}
Context: Most deaths caused by transfusion worldwide is due to transmission of infectious agents like Virus, Bacteria and Protozoa. The risk of disease transmission increases many fold if blood donor selection is inappropriate (such as blood from professional donor) and testing of all blood for transfusion is inadequate.

Methods: The study was carried out in transfusion Medicine department of Dhaka Medical College Hospital from January to December 2009 to see the prevalence of HBV, HCV, HIV, Treponema pallidum and Malarial parasite among the donor.

Results: The study was carried out on 21448 donors, among whom 4464 (20.81\%) were voluntary and 16984 (79.19\%) were relative donors. Among 21448 donors, 21081 (98.29\%) were selected and 367 (1.71\%) were rejected. Out of 21081 selected donors 4406 (20.9\%) were voluntary donor and 16675 (79.1\%) were relative donor. Out of 367 rejected 58 (15.8\%) were voluntary and 309 (84.2\%) were relative donor. On analyzing the 367 rejected cases, HBs $\mathrm{Ag}$ positive 297 (80.93\%), Anti HCV positive 38 (10.35\%), Malarial Parasite positive 23 (6.27\%), Treponema pallidum positive 9 (2.45\%) and none found positive for HIV.
\end{abstract}

Key words: Blood donor, Voluntary donor, Relative donor, Screening.

J Dhaka MedColl. 2010; 19(1) : 16-18.

\section{Introduction:}

Transfusion of blood and blood component as a specialized modality of patient management saves million of lives each year worldwide. The chance of becoming a carrier of a particular agent and the risk of transmitting the agent can be reduced by appropriate donor selection and screening. Infectious agents that are present only in blood cells (Malarial parasite) can be transmitted by all blood components except cell free plasma. Those viruses that are present in plasma (HBV, $\mathrm{HCV}$ ) can be transmitted by cell free plasma and plasma fractions as well as by cellular components. HIV is found in both mononuclear cells and free in plasma. Infections in blood donors can be transmitted to blood product recipients. Strategies to reduce the risks to blood product recipients have involved public education programs, administration of questionnaires pertaining to HIV, HBV, HCV, Treponema pallidum, Malarial parasite risk factors, the use of a confidential self-exclusion (CSE) option which allows the donor to indicate in a confidential manner that their blood should not be used for transfusion, and direct questioning about risk behaviors. These strategies have been found to eliminate some high risk and some infected units. While there have been notable decreases in HIV-positive donations some donors, who are aware of their high-risk behaviors, continue to donate without selfdeferral.

\section{Materials and Methods:}

The study was carried out on 21448 donors in Department of Transfusion Medicine, Dhaka Medical College Hospital, Dhaka from January to December 2009. The age distribution of donors was 18 to 58 years with informed

1. Assistant Registrar, Department of Transfusion Medicine, DMCH, Dhaka.

2. Associate Professor, Department of Transfusion Medicine, DMCH, Dhaka.

3. Medical Officer, Department of Transfusion Medicine, DMCH, Dhaka.

4. Assistant Professor, Department of Transfusion Medicine, DMCH, Dhaka.

5. Assistant Professor, Department of Medicine, DMCH, Dhaka.

6. Consultant (Cardiology), NITOR, Dhaka.

Correspondence : Dr. Fahmida Sharmin Chowdhury 
consent and fulfilling the criteria of a donor, blood was collected. Blood samples collected in pre labeled pilot tube during collection of blood. Serum was separated from clotted blood. Detection of HBsAg, Anti-HCV, Anti-HIV, AntiTreponema and Anti-Malaria were done by latex agglutination Immuno-chromatographic test (ICT) and reconfirmed by ELISA. All tests were performed in accordance with the instruction of reagent manufacturer. Other necessary laboratory information was collected after completion of test from the registrar of Transfusion Medicine Department of DMCH.

\section{Results:}

Results are shown in the following diagrams.

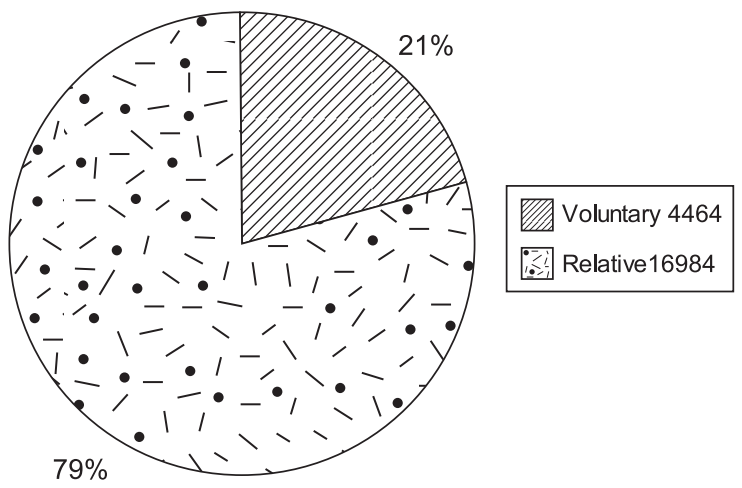

Fig.-1: Distribution by type of Donor $(n=21448)$

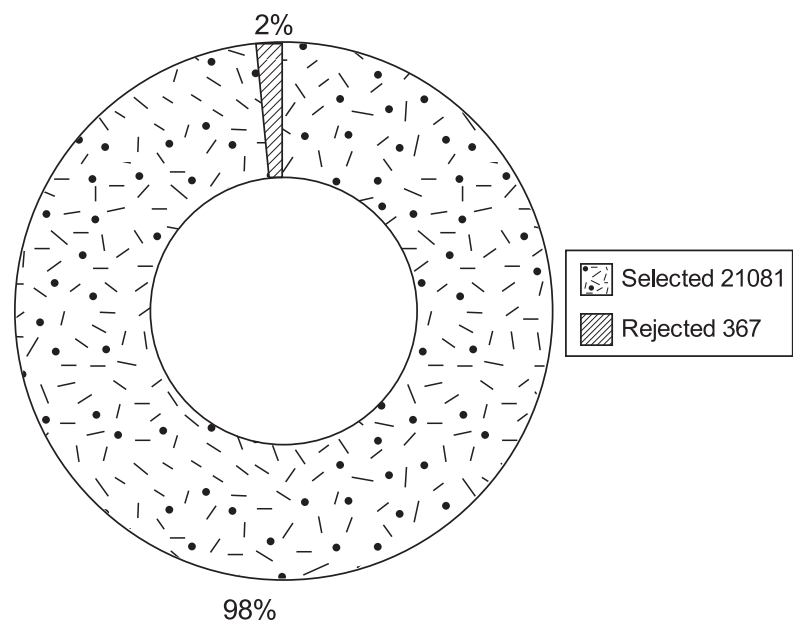

Fig.-2: Dristibution by Selection and Rejection $(n=21448)$

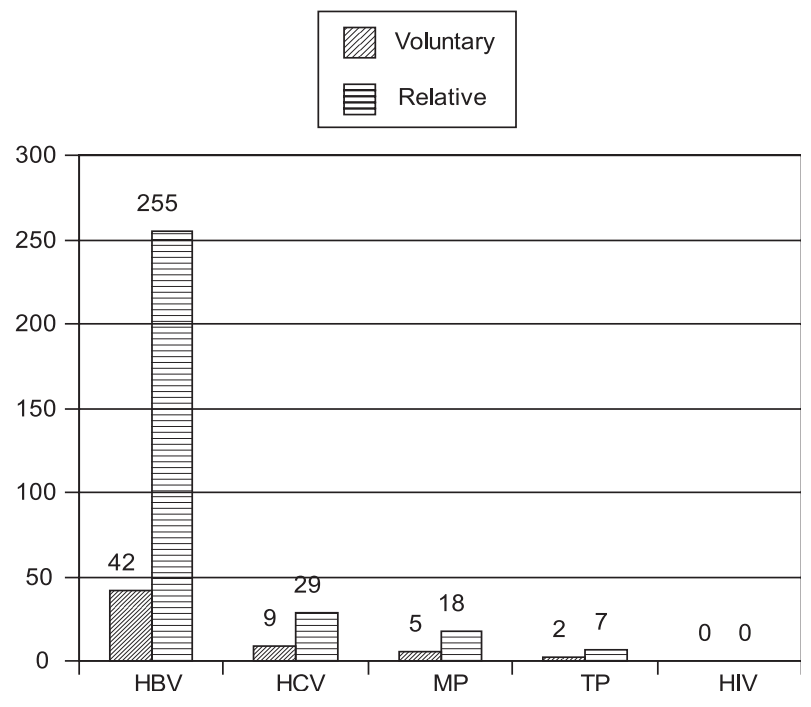

Fig.-3: Distribution by screening reactive among voluntary and relative donor.

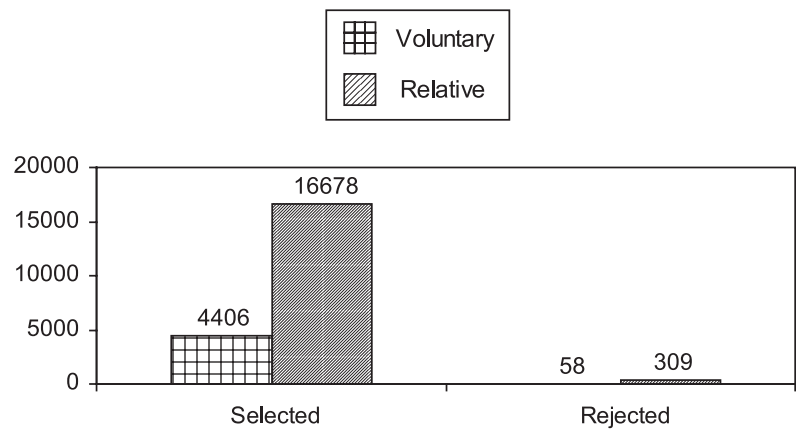

Fig.-4: Distribution by screening positive
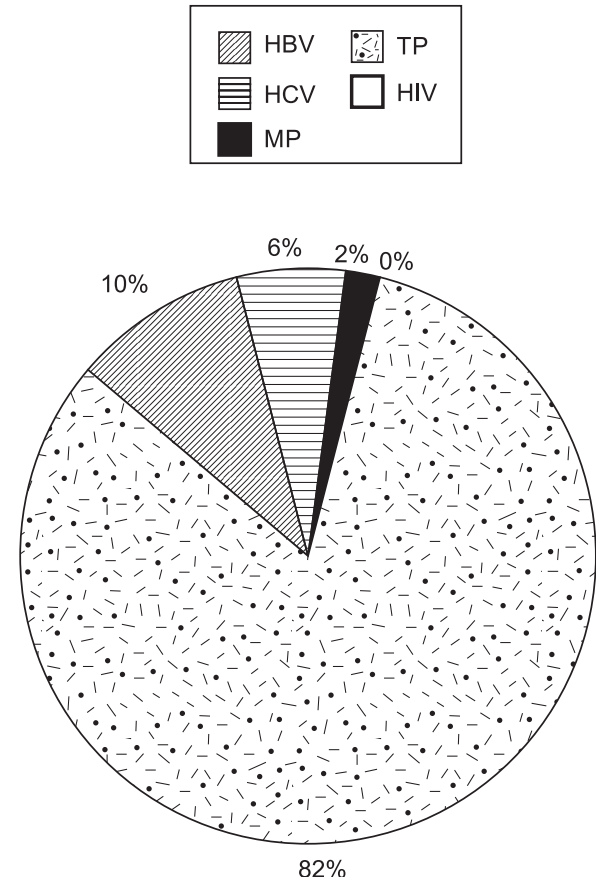

Fig.-5: Distribution by screening positive. 


\section{Discussion:}

In Transfusion Medicine Department of Dhaka Medical College Hospital, no professional donor had taken part in donation system. But the number of voluntary donor were poor, only $20.81 \%$

In this study out of 21448 donors $\mathrm{HBsAg}$ positive 297 (1.38\%), Anti HCV positive 38 (0.1\%), Malarial Parasite positive 23 (0.11\%), Treponema pallidum positive $9(0.4 \%)$.

In Bombay, $\mathrm{Hbs} A g$ positive in $6 \%$ cases and in Pakistan it is $5 \%{ }^{1}$. In one study done by Safe Blood Transfusion Center, HbsAg positive (0.96\%), HCV positive $(0.15 \%)$, HIV positive $(0.0065 \%)$ TP positive $(0.15 \%)^{2}$. Study in $\mathrm{KMCH}$, HBsAg positive (1.39\%), HCV positive $(0.024 \%)$, HIV positive $(0.38 \%)^{3}$. In a study in Canada, HBsAg positive (12.40\%), HCV positive (16.83\%), HIV positive $(0.38 \%)^{4}$. In a study in Italy HBV positive is $(4.86 \%)^{5}$. In another in Turkey, HBV positive $(1.38 \%), \operatorname{HCV}(0.35 \%)^{6}$.

\section{Conclusion:}

Voluntary donors are safer. So we should encourage voluntary donation. Mandatory screening of donated blood should be done for HBV, HCV, HIV, Treponema pallidum, malarial parasite and other transfusion transmissible diseases will ensure safe blood transfusion.

\section{Acknowledgement:}

Special thanks to all medical and non-medical persons of the Department of Transfusion Medicine for their service, work, and cordial help for the study.

\section{References:}

1. Rahman K, Khan AA, Huda $Z$, et al prevalence of seromarkers of HBV and HCV in health care personal and apparently healthy blood donors. J Pak Med Assoc. 46: 152-4.

2. Safe Blood Transfusion Center. Annual reports of donor screening, DMCH, Dhaka. 2009.

3. Ahmed MU, Begum HA, Hossain T,Incidence of common transfusion transmitted disease among blood donors. JAFMC, Bangladesh. 2009; 5(1):

4. Chiavetta JA, Escobar M, et al. Incidence of HBV, HCV and HIV in Canada, CMAJ. 2003; 169(8):

5. Manjinni $\mathrm{P}$, Guurotto $\mathrm{M}$, et al. Italian blood donors with anti-HBc and occult HBV infection. Haematologia. 2007; 92(12):

6. The prevalence of HBV,HCV and HIV infection among donor in Ijmer, Turkey. Indian $\mathrm{J}$ Med Microbiol. 26(3): 